\title{
Mathematical Solution of Two Dimensional Advection-Diffusion Equations
}

\author{
Khaled Sadek Mohamed Essa, Sawsan Ibrahim Mohamed EI Saied \\ Department of Mathematics and Theoretical Physics, NRC, Atomic Energy Authority, Cairo, Egypt \\ Email address: \\ mohamedksm56@yahoo.com (K. S. M. Essa)

\section{To cite this article:} \\ Khaled Sadek Mohamed Essa, Sawsan Ibrahim Mohamed El Saied. Mathematical Solution of Two Dimensional Advection-Diffusion \\ Equations. Journal of Chemical, Environmental and Biological Engineering. Vol. 3, No. 1, 2019, pp. 8-12. \\ doi: $10.11648 /$ j.jcebe.20190301.12
}

Received: April 8, 2019; Accepted: May 17, 2019; Published: June 12, 2019

\begin{abstract}
The Laplace conversion technique was applied to the Advection-Diffusion Equations (ADE) in two dimensions to obtain crosswind integrated normalized concentration, consider wind speed and the vertical eddy diffusivity ' $\mathrm{K}_{\mathrm{z}}$ ' are constant. Data set used from atmospheric diffusion experiments conducted in the northern part of Copenhagen, Denmark was observed for hexafluoride traceability (SF6). A comparison was made between current results, previous work results and data. One finds that the present and previous work crosswind integrated normalized concentration results are agreement well with observed data (one to one) and others lie inside the factor of two and four.
\end{abstract}

Keywords: Laplace Transforms Technique, Wind Speed, Copenhagen, Denmark, Advection-Diffusion Equations, Eddy Diffusivity

\section{Introduction}

Models of atmospheric dispersion refer to mathematics Description of the transport of pollutants into the atmosphere. The term dispersion is used in this context to describe combination of propagation (due to turbulent vortex movement) and Adhesion (due to wind) occurring within the air near Surface of the Earth [1]. Analytical solution of the equation for air diffusion depends on different forms NonGaussian solutions. Analytical solution with energy law for wind speed and vortex spread with the real assumption was examined by Demuth [2].

The atmosphere is an important route to either transport airborne pollutants or radioactive releases from nuclear power plants to the environment and thus man. It is therefore necessary to obtain sufficient information on this path in order to estimate the radioactive dispersion to the population of the region and thus be able to assess the radioactive effect on the human being.

The present guide describes the meteorological phenomena and mechanisms involved in the dispersion of the released effluents in the atmosphere, discusses methods which may be to calculate the concentration and deposition in the region specifies the data needed for input to the models.
Khaled Essa, et al. are solved the advection diffusion equation in three dimensions space $(\mathrm{x}, \mathrm{y}, \mathrm{z})$ using separation of variables technique to evaluate pollutant concentration per emission rate, taking eddy diffusivities of pollutants and mean wind speed in neutral case [3].

Gantulga Tsedendorjand, et al. are developed digital schemas based on 1 and 2 step GIRMs to evaluate the twodimensional problem of Advection-Diffusion Equations in an infinite field. Accurate approximate solutions are obtained in both cases from GIRM and compared with accurate solutions. The GIRM derivation is clear and implementation is simple [4].

Khaled Essa, et al. has been obtained the normalized integrated concentration of pollutant after solving temporally diffusion equation using the method of separation variable considering the eddy diffusivities which measuring at night or at any time in high inversion layer in the stable condition [5].

Chatterjee, et al. are solved two-dimensional AdvectionDispersion Equation (ADE) in a semi-infinite domain [6].

Its analytical/numerical solutions along with an initial condition and two boundary conditions help to understand the contaminant or pollutant concentration distribution behavior through an open medium like air, rivers, lakes and 
porous medium like aquifer, on the basis of which remedial processes to reduce or eliminate the damages may be enforced. In the initial works while obtaining the analytical solutions of dispersion problems in the ideal conditions, the basic approach was to reduce the advection-diffusion equation into a diffusion equation by eliminating the advection terms [7].

The literature presents several methods to analytically solve the partial differential equations governing transport phenomena. For example, the method of separation-ofvariables is one of the oldest and most widely used techniques. Similarly, the classical Green's function method can be applied to problems with source terms and inhomogeneous boundary conditions on finite, semi-infinite, and infinite regions [8].

In the present work, the advection diffusion equation (ADE) is solved in two directions to obtain the crosswind integrated ground level concentration and Laplace transform technique was used considering the wind speed and eddy diffusivity are constant. The used data set was observed from the atmospheric diffusion experiments conducted at the northern part of Copenhagen, Denmark for The tracer Sulfur Hexafluoride $\left(\mathrm{SF}_{6}\right)$. Comparison between the present, previous work results and data was observed

\section{Analytical Solution}

The transfer of the steady state to release non-reactive pollutants from a point source is described by following the partial differential equation:

$$
\mathrm{U} \frac{\partial \mathrm{C}}{\partial \mathrm{x}}=\frac{\partial}{\partial \mathrm{y}}\left(\mathrm{K}_{\mathrm{y}} \frac{\partial \mathrm{C}}{\partial \mathrm{y}}\right)+\frac{\partial}{\partial \mathrm{z}}\left(\mathrm{K}_{\mathrm{z}} \frac{\partial \mathrm{C}}{\partial \mathrm{z}}\right)
$$

$\mathrm{C}$ denotes the pollutant concentration, $\mathrm{K}_{\mathrm{z}}$ is the turbulent eddy diffusivity coefficient assumed to be a function of the variable $\mathrm{z}$ and $\mathrm{u}$ is the mean wind oriented in the $\mathrm{x}$ direction and a function of the variable $z$. In deriving the above equation, the $\mathrm{x}$-axis is taken in the direction of the wind speed and the transport due to diffusion in the direction of the wind is neglected compared to that due to advection.

The crosswind-integrated concentration $\left(\mathbf{C}_{\mathbf{y}}\right)$ is obtained by integrated equation (1) with respect to $y$ from $-\infty$ to $+\infty$ as follows:

$$
\mathrm{U}(\mathrm{z}) \frac{\partial \overline{\mathrm{C}}_{\mathrm{y}}}{\partial \mathrm{x}}=\mathrm{K}_{\mathrm{z}} \frac{\partial^{2} \overline{\mathrm{C}}_{\mathrm{y}}}{\partial \mathrm{Z}^{2}}
$$

Divide equation (2) on $U(\mathrm{z})$ to obtain the following:

$$
\frac{\partial \overline{\mathrm{C}}_{\mathrm{y}}}{\partial \mathrm{x}}=\frac{\mathrm{K}_{\mathrm{z}}}{\mathrm{U}} \frac{\partial^{2} \overline{\mathrm{C}}_{\mathrm{y}}}{\partial \mathrm{Z}^{2}}
$$

Equation (3) was subject to following the boundary conditions:

1-The pollutant is released from an elevated source of strength $Q$ located at $(0, \mathrm{~h})$ i.e.:

$$
\mathrm{U} \overline{\mathrm{C}}_{\mathrm{y}}(\mathrm{x}, \mathrm{z})=\mathrm{Q} \delta(\mathrm{z}-\mathrm{hs}) \text { at } \mathrm{x}=0
$$

Where "hs" is a stack height and $\delta()$ is the Dirac delta function.

2-The concentration of the pollutant tends to zero at large distance of the source i.e.:

$$
\overline{\mathrm{C}}_{\mathrm{y}}(\mathrm{x}, \mathrm{z})=0 \text { at } \mathrm{x} \rightarrow \infty, \mathrm{z}=0
$$

3- The flux at the ground can be given by:

$$
\mathrm{Kz} \frac{\partial \overline{\mathrm{C}}_{\mathrm{y}}}{\partial \mathrm{z}}=0 \text { at } \mathrm{z}=0
$$

Consider wind speed and the vertical eddy diffusivity ' $\mathrm{K}_{\mathrm{Z}}$ ' are constant

Replace equations (3) and (4) in equation (3) to get the following

$$
\frac{\partial \overline{\mathrm{C}}_{\mathrm{y}}}{\partial \mathrm{x}}=\frac{\mathrm{K}_{\mathrm{z}}}{\mathrm{U}} \frac{\partial^{2} \overline{\mathrm{C}}_{\mathrm{y}}}{\partial \mathrm{Z}^{2}}
$$

Apply the Laplace transform on equation (7) to respect $\mathrm{x}$. Obtains:

$$
\begin{gathered}
\check{\mathrm{C}}(\mathrm{s}, \mathrm{z})=\mathrm{L}_{\mathrm{p}}\{\mathrm{x}(\mathrm{s}) ; \mathrm{x} \rightarrow \mathrm{s}\} \\
\widetilde{\mathrm{sC}}_{\mathrm{y}}(\mathrm{s}, \mathrm{z}) \frac{\partial \widetilde{\overline{\mathrm{C}}_{\mathrm{y}}}(\mathrm{s}, \mathrm{z})}{\partial \mathrm{x}}-\mathrm{C}(0, \mathrm{z})=\frac{\mathrm{K}_{\mathrm{z}}}{\mathrm{U}} \frac{\partial^{2} \widetilde{\mathrm{C}_{\mathrm{y}}}(\mathrm{s}, \mathrm{z})}{\partial \mathrm{z}^{2}}
\end{gathered}
$$

Equation (8) becomes:

$$
\frac{\partial^{2} \widetilde{\overline{\mathrm{C}}_{\mathrm{y}}}(\mathrm{s}, \mathrm{z})}{\partial \mathrm{Z}^{2}}-\frac{\mathrm{U}}{\mathrm{K}_{\mathrm{z}}} \mathrm{s} \widetilde{\overline{\mathrm{C}}_{\mathrm{y}}}(\mathrm{s}, \mathrm{z})=\frac{\mathrm{U}}{\mathrm{K}_{\mathrm{z}}} \mathrm{C}(0, \mathrm{z})
$$

The solution of this equation

$$
\frac{\widetilde{\mathrm{C}_{\mathrm{y}}}(\mathrm{s}, \mathrm{z})}{\mathrm{Q}}=\frac{\mathrm{UQ} \mathrm{C}(0, \mathrm{z})}{\mathrm{K}_{\mathrm{z}}\left(\mathrm{D}-\sqrt{\frac{\mathrm{U}}{\mathrm{K}_{\mathrm{z}}} \mathrm{s}}\right)\left(\mathrm{D}+\sqrt{\frac{\mathrm{U}}{\mathrm{K}_{\mathrm{z}}} \mathrm{s}}\right)}=\frac{\mathrm{UQ} \mathrm{C}(0, \mathrm{z})}{\mathrm{K}_{\mathrm{z}}\left(\mathrm{D}-\sqrt{\frac{\mathrm{U}}{\mathrm{K}_{\mathrm{z}}}}\right)\left(\mathrm{D}+\sqrt{\frac{\mathrm{U}}{\mathrm{K}_{\mathrm{z}}} \mathrm{s}}\right)}=\left(\sqrt{\frac{\mathrm{U}}{\mathrm{K}_{\mathrm{z}}}}\left(\frac{\mathrm{e}^{-\mathrm{h} \sqrt{\frac{\mathrm{U}}{\mathrm{K}_{\mathrm{z}}}} \mathrm{s}}}{\sqrt{\mathrm{s}}}\right)\right)
$$

Where

$\frac{\mathrm{R}(\mathrm{x})}{(\mathrm{D}-\mathrm{m})}=\mathrm{e}^{\mathrm{mx}} \int \mathrm{e}^{-\mathrm{mx}} \mathrm{R}(\mathrm{x}) \mathrm{dx}$ Murray, et al. [9]

Substituted from equations (4) in equation (9) to get the following:

$$
\frac{\widetilde{\mathrm{C}_{\mathrm{y}}}(\mathrm{s}, \mathrm{z})}{\mathrm{Q}}=\sqrt{\frac{\mathrm{U}}{\mathrm{K}_{\mathrm{z}}}}\left(\frac{\mathrm{e}^{-\mathrm{h} \sqrt{\frac{\mathrm{U}}{\mathrm{K}_{\mathrm{z}}}}}}{\sqrt{\mathrm{s}}}\right)
$$

Apply the Laplace inverse transform on equation (10) to respect x. Obtains

$$
\frac{\bar{C}_{y}(x, z)}{Q}=\frac{h}{2 K_{z} \sqrt{\pi x}} e^{-\frac{U^{2} h}{4 k_{z}^{2} x}}
$$

\section{Results and Discussion}

From the atmospheric diffusion experiments conducted at 
the northern part of Copenhagen, Denmark, under unstable conditions the tracer sulfur hexafluoride $\left(\mathrm{SF}_{6}\right)$ was released from a tower at a height of $115 \mathrm{~m}$ without buoyancy was used data set was observed by Gryning, et al [10] and Gryning, et al [11]. The values of different parameters such as stability, wind speed at $115 \mathrm{~m}$, and downwind distance during the experiment was represented A comparison is made between the results calculated in this study and the results obtained from Copenhagen, Denmark and previous results. Most of the calculated results are better than the previous results and are consistent with the measured results (see Table 1). Distributed plots of concentrations observed versus projected cross-structures that have been normalized with the emission source rate. Points are found between dashed lines in two and four factors (see figure 1). Comparison of wind direction distance and integral concentrations (see Figure 2). A statistical method is displayed to confirm the results. Achieved in COR to obtain better calculated results and FAC2 (see Table 2).

Table 1. Comparison of the Predicted and observed crosswind-integrated concentrations normalized with the emission source rate.

\begin{tabular}{|c|c|c|c|c|c|c|c|}
\hline \multirow{2}{*}{ Run no. } & \multirow{2}{*}{ Stability } & \multirow{2}{*}{ Height(m) } & \multirow{2}{*}{ Wind speed $(\mathrm{m} / \mathrm{s})$} & \multirow{2}{*}{ Distance (m) } & \multicolumn{3}{|c|}{ Concentration per emission rate } \\
\hline & & & & & Observed & Predicted & Reference [13] \\
\hline 1 & very unstable & 1980 & 3.03 & 1900 & 6.48 & 4.90 & 5.5 \\
\hline 1 & Slightly unstable & 1980 & 3.03 & 3700 & 2.31 & 3.70 & 3.1 \\
\hline 2 & Slightly unstable & 1920 & 7.99 & 2100 & 5.38 & 2.10 & 3.6 \\
\hline 2 & Moderately unstable & 1920 & 7.99 & 4200 & 2.95 & 4.20 & 1.2 \\
\hline 3 & Moderately unstable & 1120 & 3.46 & 1900 & 8.2 & 1.90 & 6.2 \\
\hline 3 & Moderately unstable & 1120 & 3.46 & 3700 & 6.22 & 3.70 & 5.4 \\
\hline 3 & Slightly unstable & 1120 & 3.46 & 5400 & 4.3 & 5.40 & 3.3 \\
\hline 5 & Slightly unstable & 820 & 4.08 & 2100 & 6.72 & 2.10 & 5.8 \\
\hline 5 & Slightly unstable & 820 & 5.05 & 4200 & 5.84 & 4.20 & 3.6 \\
\hline 5 & Slightly unstable & 820 & 5.05 & 6100 & 4.97 & 6.10 & 2.3 \\
\hline 6 & Slightly unstable & 1300 & 5.05 & 2000 & 3.96 & 2.00 & 2.8 \\
\hline 6 & Slightly unstable & 1300 & 11.73 & 4200 & 2.22 & 4.20 & 1.45 \\
\hline 6 & Slightly unstable & 1300 & 11.73 & 5900 & 1.83 & 5.90 & 1.4 \\
\hline 7 & Moderately unstable & 1850 & 11.73 & 2000 & 6.7 & 2.00 & 6.4 \\
\hline 7 & Moderately unstable & 1850 & 5.91 & 4100 & 3.25 & 4.10 & 5.2 \\
\hline 7 & Moderately unstable & 1850 & 5.91 & 5300 & 2.23 & 5.30 & 2.1 \\
\hline 8 & Neutral & 810 & 5.91 & 1900 & 4.16 & 1.90 & 3.2 \\
\hline 8 & Neutral & 810 & 7.73 & 3600 & 2.02 & 3.60 & 2.01 \\
\hline 8 & Neutral & 810 & 7.73 & 5300 & 1.52 & 5.30 & 1.4 \\
\hline 9 & Slightly unstable & 2090 & 7.73 & 2100 & 4.58 & 2.10 & 2.2 \\
\hline 9 & Slightly unstable & 2090 & 8.31 & 4200 & 3.11 & 4.20 & 3 \\
\hline 9 & Slightly unstable & 2090 & 8.31 & 6000 & 2.59 & 6.00 & 1.62 \\
\hline
\end{tabular}

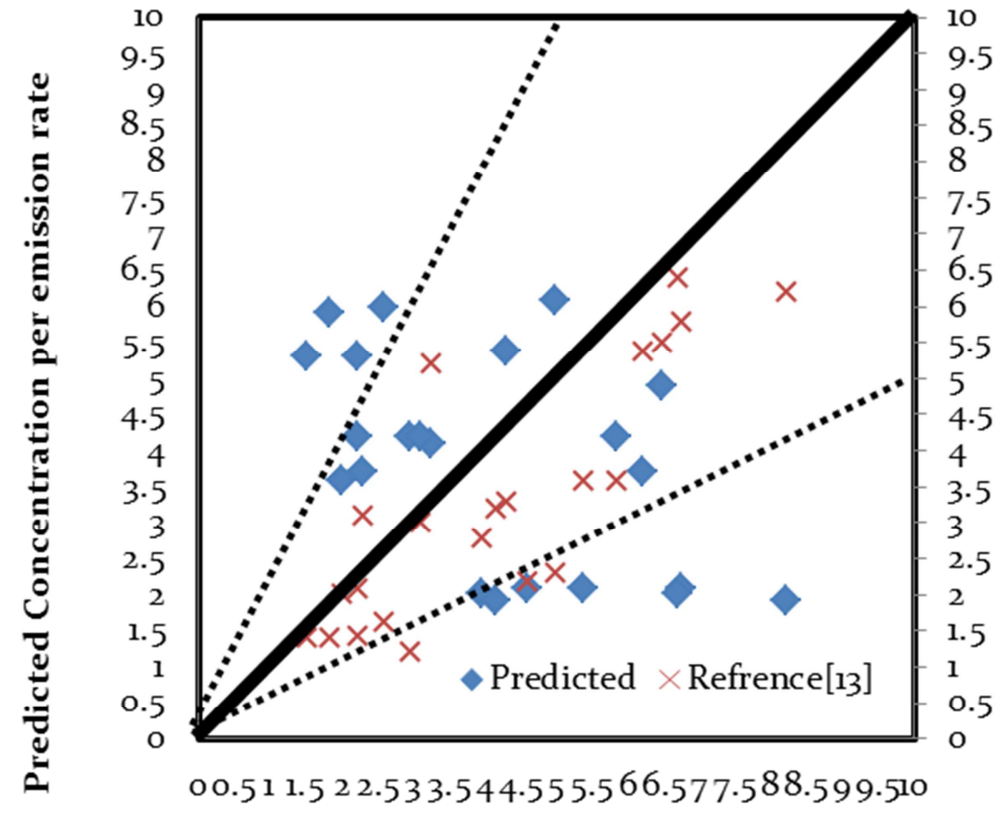

Observed Concentration per emission rate

Figure 1. A scattered plot of observed concentrations versus Predicted crosswind-integrated normalized concentrations with the emission source rate. Points between dashed lines are in factor of two and four. 


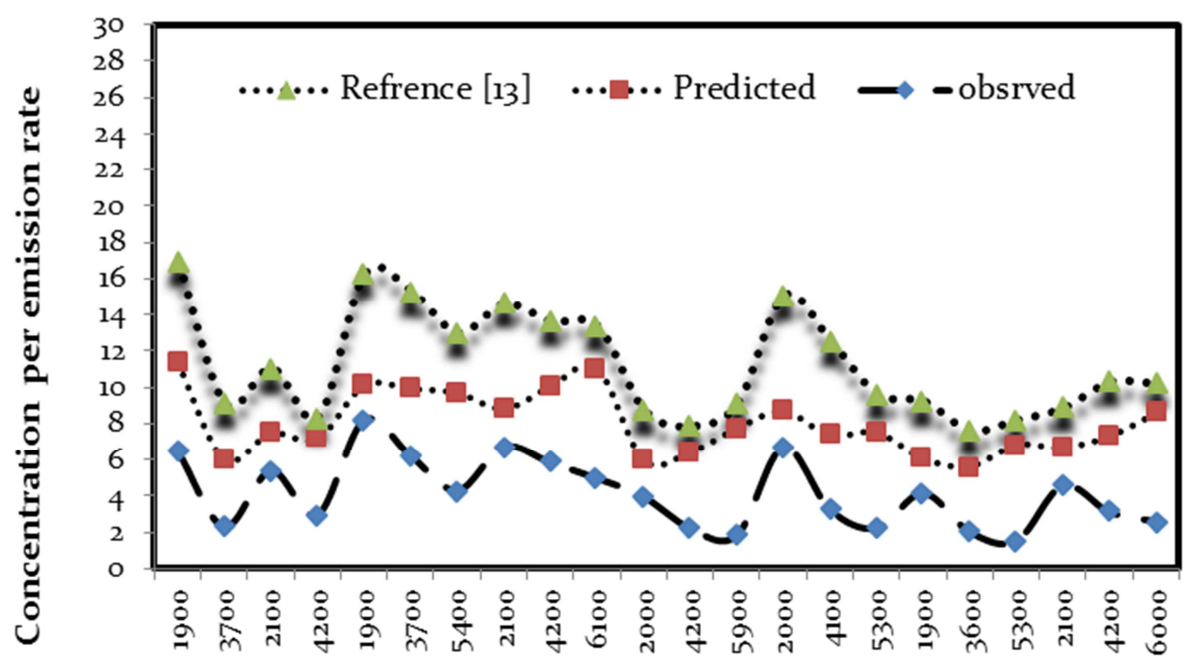

Downwind distance $(\mathrm{m})$

Figure 2. Comparison between the downwind distance and crosswind-integrated normalized concentrations.

\section{Statistical Method}

At present, the statistical method is presented and a comparison will be made between the analytical and statistical results obtained by Hanna [12].

$$
\text { Fraction Bias }(\mathrm{FB})=\frac{\left(\overline{\mathrm{C}_{\mathrm{o}}}-\overline{\mathrm{C}_{\mathrm{p}}}\right)}{\left[0.5\left(\overline{\mathrm{C}_{\mathrm{o}}}+\overline{\mathrm{C}_{\mathrm{p}}}\right)\right]}
$$

Normalized Mean Square Error $(\mathrm{NMSE})=\frac{\overline{\left(\mathrm{C}_{\mathrm{p}}-\mathrm{C}_{\mathrm{o}}\right)^{2}}}{\overline{\left(\mathrm{C}_{\mathrm{p}} \mathrm{C}_{\mathrm{o}}\right)}}$ Correlation Coefficient $(\mathrm{COR})=\frac{1}{\mathrm{~N}_{\mathrm{m}}} \sum_{\mathrm{i}=1}^{\mathrm{N}_{\mathrm{m}}}\left(\mathrm{C}_{\mathrm{pi}}-\overline{\mathrm{C}_{\mathrm{p}}}\right) \times \frac{\left(\mathrm{C}_{\mathrm{oi}}-\overline{\mathrm{C}_{\mathrm{o}}}\right)}{\left(\sigma_{\mathrm{p}} \sigma_{\mathrm{o}}\right.}$

$$
\text { Factor of Two }(\text { FAC2 })=0.5 \leq \frac{C_{p}}{C_{o}} \leq 2.0
$$

The following standard statistical performance measures are distinguished by agreement between model expectation $\left(\mathrm{Cp}=\mathrm{C}_{\mathrm{pred}} / \mathrm{Q}\right)$ and observations $\left(\mathrm{Co}=\mathrm{C}_{\mathrm{obs}} / \mathrm{Q}\right)$. Where $\sigma_{\mathrm{p}}$ and $\sigma_{o}$ are the standard deviations of $C_{p}$ and $C_{o}$ respectively.

Table 2. Statistical evaluation of models results.

\begin{tabular}{lllll}
\hline Models & NMSE & FB & COR & FAC2 \\
\hline Present & .21 & .13 & .97 & .96 \\
Reference [13] & .13 & .23 & .93 & .82 \\
\hline
\end{tabular}

\section{Conclusion}

The advection diffusion equation (ADE) is solved in two directions to obtain the crosswind integrated ground level concentration and Laplace transform technique was used considering the wind speed and eddy diffusivity are constant. The used data set was observed from the atmospheric diffusion experiments conducted at the northern part of Copenhagen,
Denmark for The tracer Sulfur Hexafluoride $\left(\mathrm{SF}_{6}\right)$. Comparison between the present, previous work and observed crosswind normalized integrated concentration are estimated. One finds that the present, previous work and observed crosswind integrated normalized concentration present data which are agreement well with observed data (one to one) and others lie inside the factor of two and factor of four.

\section{Acknowledgements}

All thanks go back to God Almighty who threatened me and helped me accomplish this work I would like to thank Dr. Khaled Sadek Mohamed Essa for his help.

\section{References}

[1] John M. (2011) The mathematical of atmospheric dispersion modeling. Society for Industrial Applied Mathematics. 53. 349-372.

[2] Demuth, C. (1978) A contribution to the analytical steady solution of the diffusion equation". Atom. Environ. 12, 1255.

[3] Khaled S. M Essa and S. E. M. Elsaid (2015) Solving the advection diffusion equation in three dimensions in neutral case. Pyrex Journal of Ecology and the Natural Environment Vol 1 (1) pp. 001-006.

[4] Gantulga Tsedendorjand Hiroshi Isshiki (2017) Numerical Solution of Two-Dimensional Advection-Diffusion Equation Using Generalized Integral Representation Method. International Journal of Computational MethodsVol. 14, No. 01.

[5] Khaled Sadek Mohamed Essa, Sawsan Ibrahim Mohamed El Saied, Ayman Marrouf (2018) Analytical Solution of Time Dependent Diffusion Equation in Stable Case. American Journal of Environmental Science and Engineering; 2 (2): 32-36.

[6] Chatterjee, A. and Singh, M. K. (2018) Two-dimensional advection -dispersion equation with depth-dependent variable source concentration. Pollution, 4 (1): 1-8. 
[7] Amruta Daga, V. H. (2013) Analytical solution of advection diffusion equation in homogeneous medium. International journal of science, spirituality, business and technology (ijssbt), vol. 2, no. 1, 2277-7261.

[8] Perez.'J. S. Guerrer. A. Pimentel. L. C. G. Skaggs. T. H. Van. Genuchten. M. Th. (2009). Analytical solution of the advection-diffusion transport equation using a change-ofvariable and integral transform technique. International Journal of Heat and Mass Transfer 52, 3297-3304.

[9] Murray R. Spiegel (1992) Advanced mathematics for engineers and scientists. McGrhill Publishing House.

[10] Gryning. S. E. and Lyck. (1984) Atmospheric dispersion from elevated sources in an urban area: Comparison between tracer experiments and model calculations. J. Climate Appl. Meteor. 23, pp. 651-660.

[11] Gryning, S. E. Holtslag, A. A. M. Irwin, J. S. Sivertsen, B. (1987) Applied dispersion modeling based on meteorological scaling parameters. Atmos. Environ. 21 (1), 79-89.

[12] Hanna, S. R. (1989). Confidence limit for air quality models as estimated by bootstrap and Jackknife resem $\neg$ bling methods. Atom. Environ. 23, 1385-1395.

[13] Tizianotirabassi, Davidson. Morerira. M. Marco. Tullio. Vilhena. Camilla. Pinto. Dam. Acosta. (2010) Comparison between non- Gaussian puff model and a model based on a time - dependent solution of advection equation. Journal of Environment, protection, and 1; 172-178. 